\title{
Confirmação da ocorrência de Loxosceles laeta (Nicolet, 1849) e Loxosceles intermedia Mello-Leitão, 1934 (Araneae; Sicariidae) no município de Pelotas, RS, Brasil
}

Everton Nei Lopes Rodrigues José EduARdo FIGUEIREDO DORNELLES

\section{Resumo}

Este trabalho trata da confirmação da ocorrência de espécies do gênero Loxosceles Heineken \& Lowe, 1832 na cidade de Pelotas, Estado do Rio Grande do Sul, Brasil. L. laeta (Nicolet, 1849) e L. intermedia Mello-Leitão, 1934 foram as espécies coletadas. L. intermedia foi a espécie mais numerosa. $\mathrm{Na}$ cidade de Pelotas, muitos espécimens foram coletados entre livros, jornais e folhas de papel. Em comparação com trabalhos prévios, algumas variações morfológicas foram observadas nestas duas espécies: em $L$. intermedia o comprimento das pernas é muito longo para o padrão da espécie; em L. laeta a mesma característica não ocorre, mas o longo comprimento do palpo dos machos é muito conspícuo. 


\section{Abstract}

This work deals the occurrence confirmation of spider species, genus Loxosceles Heineken \& Lowe, 1832, in Pelotas, Rio Grande do Sul, Brazil. L. laeta (Nicolet, 1849) and L. intermedia MelloLeitão, 1934 were the collected species. L. intermedia was the most numerous specie. In Pelotas many specimens were collected, over old books, newspapers and paper leaves. In comparasion to previous papers, some morphological variations were observed in this two species: in $L$. intermedia the length of legs is much larger than the specie general pathern; in L. laeta the same feature do not occur but the long length of male's palp is very conspicuous.

\section{Introdução}

O gênero Loxosceles Heinecken \& Lowe, 1835, inclui as popularmente conhecidas aranhas marrons, de ampla importância médica no Brasil e em outros diversos países. O gênero foi criado, baseando-se na espécie européia Loxosceles rufescens (Dufour, 1820) sendo esta praticamente cosmopolita. A inclusão desse gênero em nível de família sofreu inúmeras modificações, mas hoje esta classificada na família Sicariidae.

Até a classificação atual, ocorreram diversas modificações, SIMON (1893) classificou o gênero Loxosceles como pertencente à familia Sicariidae; mas GERTSCH (1958) inclui-o em Scytodidae; para finalizar, PLATNICK (1991) baseando-se na metodologia da sistemática filogenética, incluiu novamente o gênero Loxosceles na família Sicariidae.

Existem algumas pequenas diferenças em alguns catálogos de aranhas, mas segundo PLATNICK (2002) existem atualmente 121 espécies nesse gênero, sendo que a maioria encontra-se na América do Norte e América do Sul, logo em seguida são mais encontradas na América Central e África, e em menor quantidade na Ásia, Europa e Oceania.

Ainda conforme este autor, no Brasil, estas aranhas ocorrem em quase todo o território, registrando a ocorrência de 10 espécies, porém FISCHER (1994), registra em um trabalho anterior apenas a ocorrência de sete espécies, sugerindo uma grande divergência existente entre autores quanto à distribuição e nomenclatura destas espécies, onde ocorre dificuldade na identificação das mesmas (Figura 1). Conforme GERTSCH (1967), ocorrem no estado do RS 
quatro espécies, Loxosceles gaucho, Loxosceles hirsuta, Loxosceles intermedia e Loxosceles laeta.

\begin{tabular}{|c|c|}
\hline $\begin{array}{l}\text { Aranhas do gênero Loxosceles que ocorrem } \\
\text { no Brasil, segundo Platinick (2002) }\end{array}$ & $\begin{array}{l}\text { Aranhas do gênero Loxosceles que ocorrem } \\
\text { no Brasil, Segundo Fischer (1994) }\end{array}$ \\
\hline Loxosceles adelaida Gertsch, 1967 & Loxosceles adelaida Gertsch, 1967 \\
\hline Loxosceles amazonica Gertsch, 1967 & Loxosceles amazonica Gertsch, 1967 \\
\hline Loxosceles anomala (Mello-Leitão, 1917) & Loxosceles gaucho Getsch, 1967 \\
\hline Loxosceles gaucho Gertsch, 1967 & Loxosceles hirsuta Mello-Leitão, 1931 \\
\hline Loxosceles hirsuta Mello-Leitão, 1931 & Loxosceles intermedia Mello-Leitão, 1934 \\
\hline Loxosceles immodesta (Mello-Leitão, 1917) & Loxosceles laeta (Nicolet, 1849) \\
\hline Loxosceles intermedia Mello-Leitão, 1934 & Loxosceles similis Mocnkhaus, 1898 \\
\hline Loxosceles laeta (Nicolet, 1849) & \\
\hline Loxosceles rufescens (Dufort, 1820) * & \\
\hline Loxosceles similis Mocnkhaus, 1898 & \\
\hline
\end{tabular}

Fig.1. Quadro comparativo relacionando as espécies do gênero Loxosceles que ocorrem no Brasil, conforme Platnick (2002) e Fischer (1994).

As aranhas do gênero Loxosceles, de uma forma geral, não são agressivas, sua picada ocorre em geral quando são pressionadas dentro de alguma roupa ou calçado, ou quando são atormentadas e impedidas de fugir. Segundo NICONELLA, et al. (1997), a picada não costuma produzir dor imediata podendo, muitas vezes, até não ser percebida pelo paciente. O quadro clínico mais comum é a forma cutânea pura, evoluindo para eritema, edema endurado e dor local com ou sem flictemas, podendo ocorrer mal-estar, febre e erupção cutânea exantemática. Em casos mais graves, como no loxoscelismo cutâneo-visceral, soma-se ao quadro local, cefaléia, náuseas, vômitos, urina "cor de lavado de carne" ou mais escura 
(hemoglobinúria macroscópica) e icterícia. Ocorre comprometimento renal que pode evoluir para oligúria e anúria (insuficiência renal aguda).

Muitas das espécies deste gênero têm hábitos tanto domiciliares como peridomiciliares, construindo teias irregulares em fendas de barrancos, sob cascas de árvores, telhas e tijolos empilhados, atrás de quadros, móveis e em cantos de paredes.

Conforme EICKSTEDT (1994), no Brasil são conhecidos acidentes atribuídos às espécies Loxosceles gaucho, Loxosceles intermedia, Loxosceles laeta e Loxosceles amazonica. Na região da cidade de São Paulo, L. gaucho é a que provoca o maior número de acidentes e nos Estados do Sul $L$. intermedia e $L$. laeta são as prevalecentes, porém Loxosceles intermedia predomina nos estados do Sul e Sudeste e Loxosceles laeta ocorre em focos isolados, principalmente, no estado de SC.

Segundo NUNES et al. (2000), na cidade de Pelotas têm ocorrido inúmeros acidentes, tornando um problema de saúde pública.

Num inventário araneológico (RODRIGUES \& DREHMER, 2000) realizado neste município, foi constatado que Sicariidae é a segunda família mais numerosa. A espécie $L$. intermedia que já havia sido coletada em diversos locais do RS, mas é apenas citada para Porto Alegre e Santa Rosa, já a espécie L. laeta, foi coletada em Pelotas por C. Biezanko em 1935 e 1958 e sua ocorrência foi publicada por GERTSCH (1967). Após esta época, não há registros de $L$. laeta ter sido novamente coletada em Pelotas.

Os indivíduos deste grupo foram analisados e identificados com objetivo de conhecer melhor as espécies que compõem a comunidade de Sicariidae em Pelotas, enriquecendo desta forma o conhecimento sobre a distribuição destas aranhas nesta região do país.

\section{Materiais e métodos}

O município de Pelotas (latitude $31^{\circ} 46^{\prime} 19^{\prime \prime} \mathrm{S}$; longitude $52^{\circ} 20^{\prime} 33^{\prime \prime}$ W), localiza-se na Província Costeira sul do Rio Grande do Sul (Figura 2). Disposta em uma planície a 7 metros do nível do mar, na Encosta da Serra do Sudeste, a área desta região é de cerca de $44.000 \mathrm{~km} 2$, ou seja, $15 \%$ da superfície total do Estado do Rio Grande do Sul. Localiza-se às margens do canal São Gonçalo, que liga as lagoas dos Patos e Mirim, a 249 km da capital do estado, 
possuindo aproximadamente 323.158 habitantes distribuídos em uma área total de $1.646,63 \mathrm{~km} 2$. Na zona urbana, vivem 301.081 pessoas que se encontram em 92.274 domicílios, já na zona rural residem 22.077 habitantes (Fonte: IBGE, Base de Informações Municipais Malha Municipal Digital 1997).

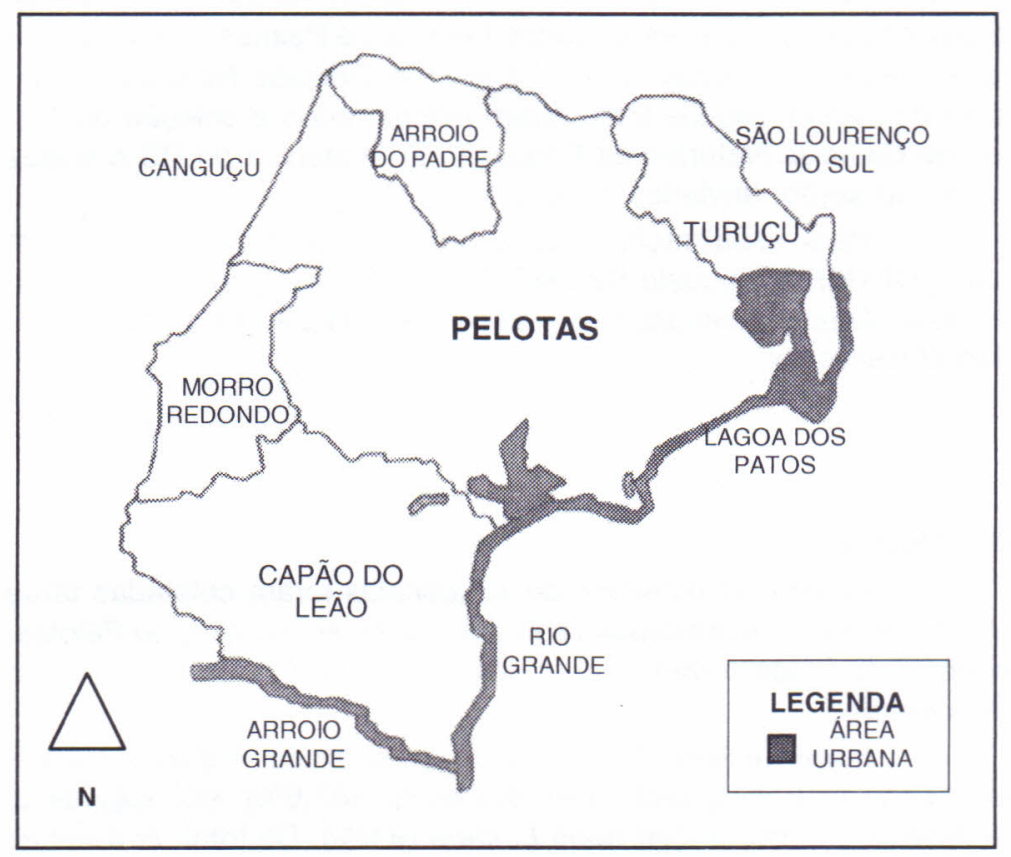

Fig. 2 - Mapa mostrando o município de Pelotas e região

As coletas ocorreram nos anos de 1999 e 2000, sendo todos os exemplares coletados em áreas urbanas, tanto em locais domiciliares quanto peridomiciliares. Foram realizadas ao acaso e manualmente, com auxílio de recipientes para apreensão. A busca deu-se em locais como teias e refúgios destas aranhas. Alguns indivíduos foram coletados pela comunidade em geral e levados até 0 laboratório.

Os exemplares foram analisados e determinados com auxílio de bibliografia especializada e comparados com material de coleções. A determinação foi feita baseando-se na morfologia dos espécimes, tanto na forma corporal quanto das genitálias das fême- 
as e nos palpos dos machos.

Para a determinação do material, foram utilizados os trabalhos de GERTSCH (1967), GERTSCH \& ENNIK (1983), MELLOLEITÃO (1934) e FISCHER (1994).

O material coletado foi catalogado, etiquetado e em seguida colocado em frascos apropriados e conservados em álcool etílico a $80 \%$. Atualmente, encontram-se depositados na coleção do Laboratório de Zoologia da Universidade Federal de Pelotas, e alguns indivíduos estão em exposição no Museu de Ciências Naturais Carlos Ritter da UFPel. Alguns lotes foram incorporados a coleção do Museu de Ciências Naturais da Fundação Zoobotânica do RS e outros foram, ou serão, enviados a especialistas.

Para visualização utilizou-se um estéreo microscópio com zoom, OLYMPUS modelo SZ4045TR. Para as fotografias, foi utilizada uma câmara fotográfica tipo reflex YASCHICA (FXD) $42-75$ mm com close-up $3 x$.

\section{Resultados}

Ambas as espécies de Loxosceles foram coletadas tanto em ambientes peridomiciliares como domiciliares, no caso de Pelotas, muitos indivíduos foram encontrados entre livros, jornais e folhas empoeiradas.

Foram examinados 92 indivíduos, entre imaturos e adultos, sendo mais freqüentes Loxosceles sp. $(67,6 \%)$, em seguida $L$. intermedia $(22,8 \%)$ e logo após L. laeta $(9,6 \%)$. Do total de aranhas coletadas, $11,5 \%$ foram machos, $25 \%$ fêmeas e $63,5 \%$ jovens, sendo estes, de difícil determinação a nível taxonômico específico, ficando apenas determinados como Loxosceles sp. (Figura 3).

O tamanho corporal médio, de ambas as espécies, ficou em 8,09 mm. Em Loxosceles intermedia o tamanho médio das fêmeas adultas foi de $10,5 \mathrm{~mm}$ e dos machos $9,5 \mathrm{~mm}$. Já na espécie Loxosceles laeta as fêmeas $11,2 \mathrm{~mm}$ e os machos $8,5 \mathrm{~mm}$.

Nos exemplares analisados neste trabalho, foram observadas algumas pequenas diferenças morfológicas para $L$. laeta e $L$. intermedia, em relação às citadas por FISCHER (1994) e GERTSCH (1967).

A coloração de $L$. intermedia foi um padrão mais escuro do que na maioria dos casos citados (Fig. 9). O macho desta espécie 
(Fig. 4), possui os apêndices locomotores maiores do que os encontrados na literatura, com uma média para os quatro pares de pernas de $27,8 \mathrm{~mm}$ de comprimento.

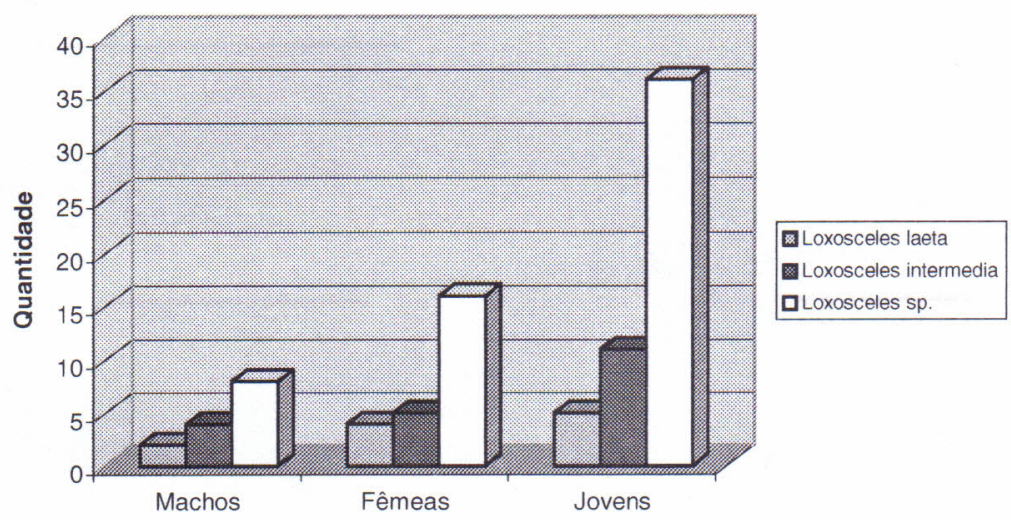

Fig. 3 - Quantidade de adultos (machos e fêmeas) e jovens das espécies loxosceles coletadas no município de Pelotas, RS

As fêmeas apresentaram dois receptáculos bem separados, com algumas variações (Figuras 5-6), sendo sempre enovelados e apresentando tubo acessório na seção basal.

Quanto à fórmula das pernas, ocorreu um predomínio nas fêmeas de 2413 e algumas com 2143, já os machos todos com 2143.

Já a espécie L. laeta, a coloração predominante em ambos os sexos foi o amarelo, mas bem claro; nos machos na região cefálica, anteriormente, a figura característica da espécie com a forma triangular e margem posterior pontiaguda, foi encontrada com uma tonalidade bem escura (Fig. 10). O palpo dos machos desta espécie, também foi encontrado com o tamanho superior à média, onde o mesmo possuiu $9,30 \mathrm{~mm}$ de comprimento (Fig. 7). A fêmea apresentou a estrutura genital padrão da espécie (Fig. 8).

Todos os indivíduos da espécie L. laeta, tanto machos quanto fêmeas, com fórmula das pernas 4213 . 

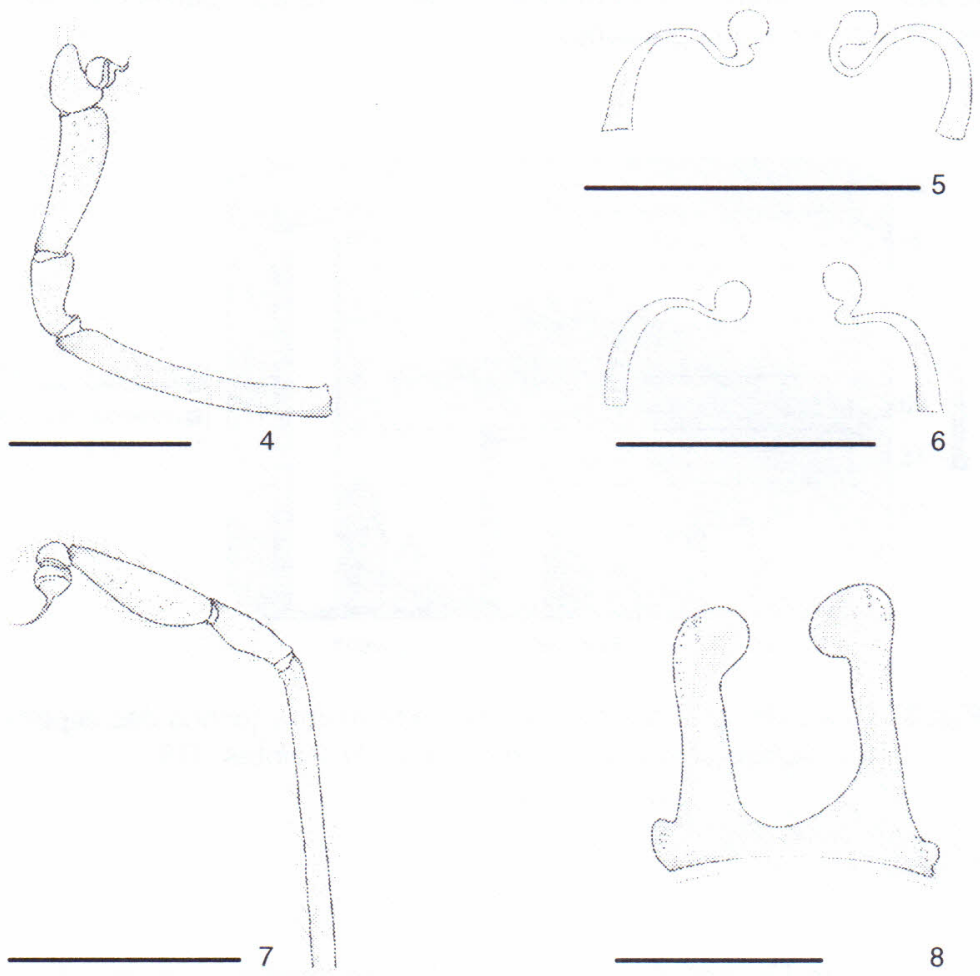

Fig. 4-6 - Loxosceles intermedia, palpo e receptáculos de duas fêmeas. 4. Palpo do macho, vista retrolateral, escala: $2 \mathrm{~mm}$. 5 . Visão ventral dos receptáculos seminais, escala: $1 \mathrm{~mm}$. 6. Visão ventral de outro receptáculo, escala: $1 \mathrm{~mm}$.

Fig. 7-8 - L. laeta, palpo e receptáculos seminais. 7. Palpo, visão retrolateral, escala: $3 \mathrm{~mm}$. 8. Receptáculo, ventral, escala: 0,5 $\mathrm{mm}$ (Desenhos do autor). 
Figuras 9 - 10. Espécies de Loxosceles (Sicariidae) encontradas no município de Pelotas, RS, Brasil (Fotos dos autores).

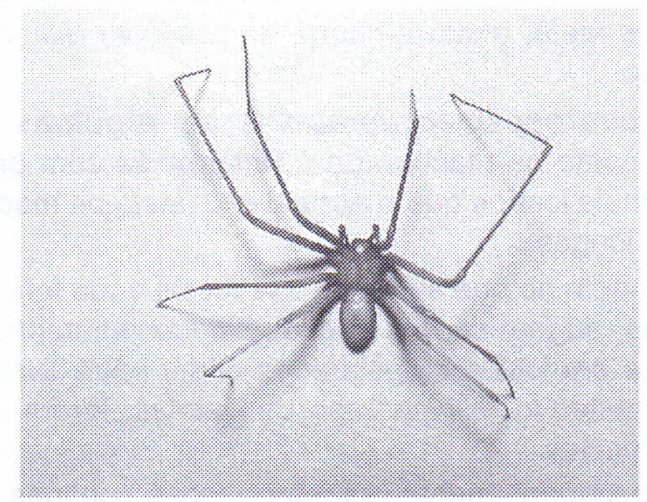

Fig. 9 - Loxosceles intermedia - Visão dorsal, fêmea. Escala: $5 \mathrm{~mm}$

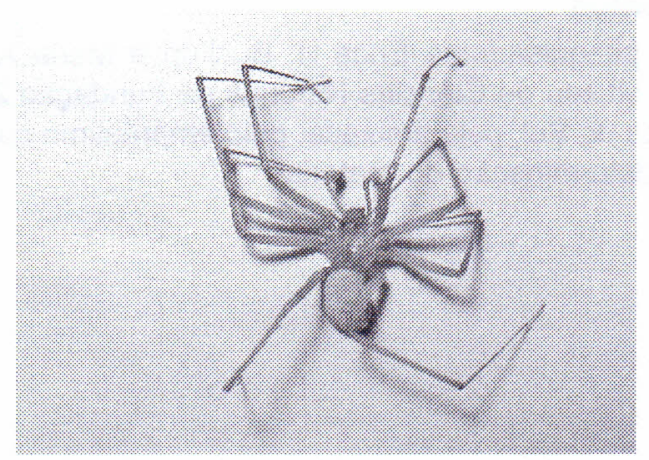

Fig. 10 - Loxosceles laeta - Visão dorsal, macho.

Escala: $4,5 \mathrm{~mm}$ 


\section{Conclusão}

Neste trabalho, confirma-se a ocorrência das espécies, Loxosceles laeta e Loxosceles intermedia, para o município de Pelotas, ocorrência que até o momento, manifestava-se pouco precisa com relação às espécies encontradas neste município, prevalecendo numericamente Loxosceles intermedia e em menor quantidade Loxosceles laeta, muito embora, ambas com distribuição geral pelo município.

Ambas espécies apresentaram algumas diferenças morfológicas como os machos de $L$. intermedia com os apêndices locomotores mais longos que o normal e $L$. laeta os machos com os palpos muito longos.

Também no que se refere aos locais onde foram coletadas estas aranhas, sugere-se que a comunidade mantenha o cuidado principalmente em locais empoeirados como entre livros, jornais e folhas, pois nestes locais, no caso de Pelotas, foram encontradas ambas as espécies

Conseqüentemente, as informações contidas neste estudo, vem a corroborar e servir de base para a realização de estudos mais complexos sobre a comunidade de aranhas que habitam as regiões urbanas e antrópicas, tanto em ambientes domiciliares ou peridomiciliares, nestes locais.

\section{Agradecimentos}

As pesquisadoras Erica H. Buckup e Maria Aparecida L. Marques, do Museu de Ciências Naturais da Fundação Zoobotânica do Rio Grande do Sul, pela amizade, pelo empréstimo da bibliografia e auxílio na determinação do material. 


\section{Referências bibliográficas}

EICKSTEDT, V. R. 1994. Aranhas de importância médica do Brasil. In Barraviera, B. (coordenador) Venenos animais: uma visão integrada. Rio de Janeiro, Ed. de Publicações Científicas - EPUC, p.151-172.

FISCHER, M. L. 1994. Levantamento das espécies do gênero Loxosceles Heinecken \& Lowe, 1832 no município de Curitiba, Paraná, Brasil. Estudos de Biologia, Paraná, vol. 03, n. 38, p. 63-88. GERTSCH, W. J. 1958. The spider genus Loxosceles in North America, Central America, and the West Indies. Am. Mus. Novit. 1907: 1-46.

GERTSCH, W. J. 1967. The spiders of genus Loxosceles in South America (Araneae, Scytodidae). Bull. Am. Mus. Nat. Hist., New York, v.136, n. 3, p. 119-173.

GERTSCH, W. J. \& ENNIK, F. 1983. The spiders genus Loxosceles in North america, and the west indies (Araneae, Loxoscelidae). Bull. Am. Mus. Nat. Hist., New York, v.175, n. 3, p. 265-359.

MELLO-LEITÃO, C. 1934. Espécies brasileiras do gênero Loxosceles. Ann. da Acad. Brasileira de Ciências, Rio de Janeiro, v.6, n.2, p. 6973.

NICONELLA, A.; BARROS, E.; TORRES, J.B. \& MARQUES, M. G. 1997. Acidentes com animais peçonhentos: consulta rápida. Porto Alegre, Hospital de Clínicas de Porto Alegre Ed. 207 p.

NUNES, A. M.; VIANNA, A. D.; RIBEIRO, P. B. \& VIANNA, E. E. S. 2000. Levantamento de acidentes com animais peçonhentos, no período de janeiro a julho de 2000 , em Pelotas, RS. In: IX CONGRESSO DE INICIAÇÃO CIENTífICA, Pelotas, RS. 2000. Resumos, V. 1, UFPel, p.215.

PLATNICK, N. I. 2002. The World Spiders Catalog - Version 2.5. [on line]. (New York). http://research.amnh.org/entomology/spiders/catalog8187/index.html (12/09/2002)

PLATNICK, N. I., J. A. CODDINGTON, R. R. FORSTER \& C. E. GRISWOLD. 1991. Spinneret morphology and the phylogeny of haplogyne spiders (Araneae, Araneomorphae). Am. Mus. Novit. 3016: 1-73.

RODRIGUES, E. N. L. \& DREHMER, C. J. 2000. Levantamento araneológico das áreas domiciliares e peridomiciliares da região urbana do município de Pelotas, RS, Brasil. In: IX CONGRESSO DE INICIAÇÃO CIENTÍfICA, Pelotas, RS. 2000. Resumos, V. 1, UFPel,.202.

SIMON, E. 1893. Histoire naturelle das araignées. Paris, 1: 257488. 
Everton Nei Lopes Rodrigues

Curso de Ciências Biológicas da UFPel enlrodrigues@yahoo.com.br José Eduardo Figueiredo Dornelles Professor Dr. Adjunto II jefdornelles@ hotmail.com Lab. de Zoologia, Departamento de Zoologia \& Genética, IB, Universidade Federal de Pelotas Pelotas, RS - Brasil 